\title{
USAGE OF WEB MAPPING SYSTEMS AND SERVICES FOR INFORMATION SUPPORT OF REGIONAL MANAGEMENT
}

\author{
Nicolay Shaparev ${ }^{1,2}$, Oleg Yakubailik ${ }^{1,{ }^{*}}$ \\ ${ }^{1}$ Institute of Computational Modeling SB RAS, 660036, Krasnoyarsk, Russia \\ ${ }^{2}$ National Research Tomsk State University, 634050, Tomsk, Russia
}

\begin{abstract}
The work considers information and computing technologies supporting regional decisions making and based on geoinformation websystems and mapping web-services. The use of such systems for the information support of regional management is now becoming common. Long-term strategic forecasting and planning of territories development, solution of various institutional and sectoral problems today are often based on the use of integrated information and computing environment, complex information systems of regional management, which are based on geospatial (mapping) data. This paper discusses technologies and webservices used in the creation and implementation of regional geoinformation web-systems. Such systems provide access to huge arrays of geospatial information and services distributed in the Internet, remote data processing with high performance multi-user computers. Problems of choosing basic software such as geoinformation platform, advantages and disadvantages of existing solutions are discussed. The software structure and basic web-GIS components are analyzed. Examples of completed projects are given.
\end{abstract}

\section{Geoinformation platform selecting}

Modern web-GIS are built according to so-called service-oriented architecture, and they can be regarded as a set of interrelated software components for spatial data operations - its import/export, cataloging, visualizing, creating, processing, distribution, etc. The technological basis for these solutions is a set of program interface libraries such as Google Maps API, Mapserver Mapscript, Scanex GeoMixer, etc.; they provide access to the functions and context of web pages mapping elements - map visualization, thematic data, for example, the road network with data on traffic, terrain, map points, catalogues of organizations, products and services, satellite images, etc [1]. High degree of the user interface interactivity, very fast regeneration of images made of small caching raster fragments (tiles), almost instant response of the move (shift) and map scale change tools and other features of spatial data visualization and management made the mapping web

Corresponding author: oleg@icm.krasn.ru 
applications very popular. Support of geodata exchange standards - KML, GML, WMS, etc. is also important $[2,3]$.

Implemented information systems are usually based on the development of specialized software tools of such kind $[4,5]$.

It is worth to pay attention to the problem of GIS platform selection existing today software based on commercial products or open (free) software [6]. Leaving financial, philosophical and situational aspects of the choice aside, we would like to note that there is often no opposition between the two approaches in today's practice. The reason is that currently available commercial and free GIS complement each other well due to data formats compatibility, data exchange standards based on web services, etc. For example, one can perform the spatial data analysis using ESRI ArcGIS, convert it with MapInfo to transfer to the customer and use free Mapserver software for presentation in the web pages, and the spatial metadata catalog can be formed by the means of GeoNetwork Open Source. At the same time, PostgreSQL open database with the PostGIS extension module can be used for the spatial data storage being almost as good in performance and functionality as the leader of commercial databases Oracle with an extension Oracle Spatial for working with spatial data for a vast majority of tasks.

The open architecture and free basic software allows to quickly implement almost any new (additional) functions in the created system that can be impossible while using expensive commercial software [7]. An additional bonus is the ability of integration with any third-party commercial products if necessary. For example, if the customer already has and uses the Oracle database - you can configure the spatial data storage subsystem of the created complex for the use of this database. If it is absent, you can install and use free software of PostgreSQL databases [8].

The most popular at the moment concept of building mapping web applications involves the creation of an interconnected web services components set, the implementation of which is carried out simultaneously on the server and the client computer (multi-tier architecture), as well as formation of a spatial data set usually made in the format of popular GIS or using a specialized geospatial database [9]. The initial geodata preparation for the web application is usually carried out outside the considered web-system; for this, standard desktop GIS are typically used today (MapInfo, ArcGIS, QGIS and others) [10].

\section{Software structure}

Using the experience of completed developments, we try to formulate programtechnological web-systems features focused on regional management tasks solution from the functional point of view [11]-

- The system should consist of client and server parts, thereby realizing the "clientserver" technology. Application of the MVC (model-view-controller) design pattern as the server part basis provides ample opportunities for the tasks solution. Using of this architecture assumes separation of the application data, user interface and control logic into three separate components. Modification of each component can be performed independently. Apart from the standard MVC elements, key units of the system are also page presentation templates and AJAX-handlers [12].

- The system geospatial data is recorded in the resource catalogue. The web application communication with the resource catalogue is conducted on the basis of serviceoriented architecture using the SOAP/XML protocol. It creates a set of functions available in the form of application programming interface (API), which offers the possibility of resources searching, filtering, management, editing, copying, moving, etc. [13] 
- Two API sets are formed: common (client) interface for user applications and advanced (server) interface for applications having the ability to control the resources catalogue, its objects and relationships between objects.

Information support for the applied regional geoinformation web-systems consists of several types of geospatial and other data and appropriate tools of their processing [14, 15]:

- tabular data stored in the database - it provides the business logic of the system, its basic content;

- thematic spatial data related to direct content of the application system objectives and functions. This type of data can be created both within the applied system user interface (based on the web browser or Windows application) and by tools of thirdparty applications - various GIS/CAD programs, etc.;

- mapping background - information resources of auxiliary character maps and satellite images used for the thematic spatial data visualizing. Own data sets can be used as well as third-party services provided by Yandex, Google, 2GIS and other companies;

- tools for creating and managing the spatial metadata and related services of visualization, search and filtering [16];

- means of geospatial data classifying - standardized classification system enables multiple data using for various projects [17];

- tools for the geospatial data web publication managing - user interfaces for the formation of geoportal thematic sections based on the web-content management system;

- tools for the systems integration, information exchange and interaction based on the geoportal information-analytical and mapping services [18].

\section{Basic web-GIS components}

Analysis of existing approaches and trends, experience of own developments in this area have allowed formulating some backbone "universal" components for the considered class of information systems [19]:

- Archiving subsystem for the basic geospatial data.

It must organize the data storage and management, tools for its loading and deleting, backup, etc. It shall be possible to register external databases in the archive including the spatial ones and organizing transparent access to them through the single software interface - i.e., the user who receives the data from the geospatial archives is not required to know where they come from - from SHP-file hosted on the same server or via connection to a third-party server (ArcGIS Server and analogues).

- Applied mapping web-services system.

It is supposed to create a set of different tools for data accessing and organization of queries to them including the ones based on standard protocols/interfaces, such as widely used open OGC Consortium standards - mapping web-services WMS, WFS, etc. Also, there should be possibility of organizing intensive computations on the server side - today, several alternative solutions exist in this field - WPS (Web Processing Service) by the OGC Consortium and others [20,21].

- Spatial metadata management subsystem.

The subsystem is designed to search and navigate across the available spatial data including the usage of spatial queries. The subsystem must provide the ability to work with different data classificators, be compatible with the existing metadata standards, allow the appropriate import/export. The subsystem base - metadata catalogue - should provide the entire range of metadata control/management. Deep integration with all other components of the applied geoinformation system is assumed [22]. 
- Web-application - a user interface to the data and its processing means.

Modern program and technological solutions are most often based on the webtechnologies of content management system (web-site). In the centralized web interface, tools for the spatial and other data search and navigation including the spatial queries usage should be developed. Information visualization services must provide the user with the data in text, mapping and tabular form. The subsystem must provide the ability to work with different classificators, information presentation forms using customizable templates, etc. Uploading and downloading of different data types shall be provided. All information should be provided on the basis of permissions, user interface personalization is desirable.

- Auxiliary services subsystem.

The modular architecture of the information system for regional management creates the need for strict formal specification of information exchange between its components. In this context, a set of web-services is necessary as a tool for the data transmission/reception in the system and between its constituent parts. For example, the "Web-portal" module forms a query for more information to the "Spatial metadata catalog" subsystem with its user interface. Next, the "Spatial metadata catalog" subsystem addresses the "Geodata archive" module for a list of specific resources. Restrictions on the access to administrative services are related to the fact that they can change a lot of things including the system breaking as a whole (if misused). That is why access to them is often limited by developers.

- Public applied services subsystem.

Unlike the above-mentioned administrative services, these services are publicly available. They provide the performance of different users' queries to the data stored in the system of regional management information support. Technologically, they are similar to the administrative ones, but they cannot break the system. Examples of services are different thematic information visualizing operations used in the formation of the information web-portal pages.

\section{Examples of implementation}

On the basis of the above principles and approaches to the creation of applied systems for regional management, works on the design, development, implementation and operation of several geoinformation web-systems were carried out. The service-oriented architecture provided good replicability of the software components, high speed of development and implementation. In most cases, the created geoinformation web-system modules were tightly integrated into the existing corporate information infrastructure [23].

The developed system of applied high-level services successfully established itself as a software technological basis of information analytical systems for regional territory and municipal authorities. A number of such systems were implemented, such as decision support systems for local government on health, education, transport and disaster management; environmental monitoring and assessment of the state of the environment; support system for socio-economic development of the region. In general, during the last five years it was implemented more than 10 analytical applications. Customers of these systems are the departments of the government of the Krasnoyarsk region, one of federal subjects of Russia. A few more development was established within the framework of the scientific research grants.

For example, the initial data for the Krasnoyarsk region fire situation-mapping interface is constantly real time updated departmental database on fires in the region. To ensure the operative data presentation in the geoinformation system, a special web-service providing the necessary pre-processing and raw data aggregation was developed. There are a number of similar system around the world [24]. 
The web-GIS can provide access to the spatial analysis for users who are not technical experts in GIS. It is very attractive to see your data in a spatial representation without using any complicated programs and being limited only by a standard web browser. An illustration to this idea is the experience of web-GIS using for a variety of information and analytical tasks by the Krasnoyarsk Region Veterinary Supervision Service.

\section{Conclusions}

The developed software and technological solutions have successfully proven themselves as a basis for resource intensive information and analytical systems of regional level for various problems solution - sectoral management information support (in the sphere of health, education, transport), environmental monitoring and the environment state assessment, forecasting of the region's socio-economic development, centralized map data information supplying.

The modular architecture of these systems, the use of standard web-services to exchange the data between these modules provide rapid adaptation of the existing software to customer's requirements, replication of individual components, their complementarity. In turn, the registration of created resources - spatial data - in the geoportal centralized catalog provides the possibility of its simultaneous use in multiple developments. This service-oriented approach based on active web technologies implementation in the applied information systems is increasingly used today.

\section{Acknowledgments}

The work was supported by grant no. 16-47-240517 from Russian Foundation for Basic Research and Krasnoyarsk Regional Fund of Support of Scientific and Scientific-technical activities.

\section{References}

[1] K. Patroumpas, N. Georgomanolis, T. Stratiotis, M. Alexakis, S. Athanasiou, Journal of Web Semantics 35, 53 (2015) doi: 10.1016/j.websem.2015.09.003

[2] O.E. Yakubailik, V.G. Popov, Computational Technologies 6, 116 (2009)

[3] H. Wu, Z. Li, H. Zhang, C. Yang, S. Shen, Computers \& Geosciences 37, 485 (2011) doi: 10.1016/j.cageo.2010.05.026

[4] A. Vyas and S. John, MATEC Web of Conferences 57, 05002 (2016) doi : 10.1051/matecconf/20165705002

[5] X. Jin, D. Guo, S. Wang, X. Zhao, Y. Song, A. Liu, W. Jiang, R. Wang and X. Li, $\begin{array}{lllll}\text { MATEC Web of } & \text { Conferences } & & \end{array}$ doi : $10.1051 /$ matecconf $/ 20152204027$

[6] A.A. Kadochnikov, O.E. Yakubailik, NSU Journal of Information Technologies 1, 37 (2015)

[7] L. Bastin, G. Buchanan, A. Beresford, J.-F. Pekel, G. Dubois, Ecological Informatics 14, 9 (2013) doi: 10.1016/j.ecoinf.2012.11.013

[8] B. R. Pickard, J. Daniel, M. Mehaffey, L. E. Jackson, A. Neale, Ecosystem Services 14, 45 (2015) doi: 10.1016/j.ecoser.2015.04.005

[9] V. Rautenbach, S. Coetzee, A. Iwaniak, Computers, Environment and Urban Systems 37, 107 (2013) doi: 10.1016/j.compenvurbsys.2012.08.001

[10] C. Heipke, ISPRS Journal of Photogrammetry and Remote Sensing 65, 550 (2010) doi: 10.1016/j.isprsjprs.2010.06.005 
[11] O.E. Yakubailik, A.A. Kadochnikov, V.G. Popov, A.A. Tokarev, Vestnik of SibGAU 25, 61 (2009)

[12] K. Zhang, B. Qu and X. Chi, MATEC Web of Conferences 31, 17002 (2015) doi : 10.1051/matecconf/20153117002

[13] R. Béjar, M. Á. Latre, F. J. Lopez-Pellicer, J. Nogueras-Iso, F.J. Zarazaga-Soria, P.R. Muro-Medrano, Computers \& Geosciences 46, $66 \quad$ (2012) doi: 10.1016/j.cageo.2012.04.010

[14] L. Xue, Y. Zhu, Y. Xue, Mathematical and Computer Modelling 58, 480 (2013) doi: 10.1016/j.mcm.2011.11.002

[15] C. Farcy, B. de Terwangne, P. Blerot, Computers and Electronics in Agriculture 47, 207 (2005) doi: 10.1016/j.compag.2004.12.005

[16] L. Díaz, C. Granell, J. Huerta, M. Gould, Applied Geography 35, 448 (2012) doi: 10.1016/j.apgeog.2012.09.008

[17] Theodor Foerster, Lassi Lehto, Tapani Sarjakoski, L. Tiina Sarjakoski, Jantien Stoter, Computers, Environment and Urban Systems 34, 79 (2010)

[18] D. J. Maguire, P. A. Longley, Computers, Environment and Urban Systems 29, 3 (2005) doi: 10.1016/j.compenvurbsys.2004.05.012

[19] O. Yakubailik, A. Kadochnikov, A. Tokarev, International Multidisciplinary Scientific GeoConference Surveying Geology and Mining Ecology Management (SGEM) 1, 487 (2015)

[20] A.A. Kadochnikov, InterCarto 20, 188 (2014)

[21] J.D. Blower, A.L. Gemmell, G.H. Griffiths, K. Haines, A. Santokhee, X. Yang, $\begin{array}{llllll}\text { Environmental Modelling \& Software 47, } 218 \quad \text { (2013) } & \text { 4 }\end{array}$ doi: $10.1016 /$ j.envsoft.2013.04.002

[22] A.G. Matveev, O.E. Yakubailik, Vestnik of SibGAU 2, 48 (2012)

[23] H.C. Guo, L. Liu, G.H. Huang, G.A. Fuller, R. Zou, Y.Y. Yin, Journal of Environmental Management 61, 93 (2001) doi: 10.1006/jema.2000.0400

[24] K. Kalabokidis, N. Athanasis, F. Gagliardi, F. Karayiannis, P. Palaiologou, S. Parastatidis, C. Vasilakos, Ecological Informatics 16, $62 \quad$ (2013) doi: 10.1016/j.ecoinf.2013.04.007 